\title{
Indefinite LQ Problem for Irregular Singular Systems
}

\author{
Qingxiang Fang, ${ }^{1,2,3}$ Jigen Peng, ${ }^{1}$ and Feilong $\mathrm{Cao}^{2}$ \\ ${ }^{1}$ School of Mathematics and Statistics, Xian Jiaotong University, Xian 710049, China \\ ${ }^{2}$ College of Science, China Jiliang University, Hangzhou 310018, China \\ ${ }^{3}$ Faculty of Science, Engineering and Technology, Swinburne University of Technology, Hawthorn, VIC 3122, Australia
}

Correspondence should be addressed to Qingxiang Fang; fangqx@cjlu.edu.cn

Received 7 August 2014; Accepted 8 October 2014; Published 16 October 2014

Academic Editor: Chunyu Yang

Copyright (C) 2014 Qingxiang Fang et al. This is an open access article distributed under the Creative Commons Attribution License, which permits unrestricted use, distribution, and reproduction in any medium, provided the original work is properly cited.

\begin{abstract}
The indefinite LQ problem for irregular singular systems is investigated. Under some general conditions, the optimal control-state pair is obtained by solving an algebraic Riccati equation. The optimal control is synthesized as state feedback. All the finite poles of the closed-loop system are located on the left-half complex plane. An example is given to show the validity of the proposed conclusion.
\end{abstract}

\section{Introduction}

Singular systems have comprehensive practical background [1-3]. Great progress has been made in the theory and its applications since 1970s [4-8]. The treatment of the LQ problem for linear systems has been well studied since the early 1960s. A detailed discussion of this topic can be found in $[9,10]$. For linear singular systems, there have also been a lot of excellent results about the LQ problem [11-13]. A linear quadratic control problem is indefinite when the cost weighting matrices for the state and the control are allowed to be indefinite. Although the indefinite LQ problem is meaningless for standard state space systems, it is solvable for singular systems under some conditions. To the best of our knowledge, [14] is the first article which studied a nonstandard quadratic regulator on a finite time horizon with indefinite weighting matrix for the control. Up to now, indefinite LQ theory has been extensively developed and has found interesting applications in finance, especially in dynamic macroeconomics theory $[15,16]$. Many applications for this problem can be seen in $[17,18]$ and the references therein.

A lot of literature about indefinite LQ control problem can be found. Trentelman [19] deals with the freeendpoint regular linear quadratic problem with indefinite cost functional in the context of linear quadratic optimal control and establishes necessary and sufficient conditions for the existence of optimal controls. Ran and Trentelman [20] consider the discrete time infinite-horizon linear quadratic problem with indefinite cost criterion and obtain necessary and sufficient conditions for the existences of optimal controls based on a geometric characterization of the set of all Hermitian solutions of the discrete time algebraic Riccati equation. Bucci and Pandolfi [21] study the finite horizon nonstandard LQ problem for an abstract dynamic system and provide necessary and sufficient conditions for finiteness of the value function corresponding to the control problem. Cui and Zhang [22] discuss the finite time horizon indefinite LQ problem for singular linear discrete time-varying systems and show that the indefinite LQ problem is dual to that of projection for backward stochastic systems. Cui et al. [23] study the indefinite LQ problem for singular discrete timevarying linear systems with multiple input delays and obtain an explicit controller by computing the gain of the smoothing estimation of dual systems. The above discussions were inducted in the premise that the singular system is regular. The indefinite LQ problem for irregular singular systems was rarely mentioned.

In this paper, the indefinite LQ problem for irregular singular systems is discussed. It is shown that the indefinite LQ problem for irregular singular systems can be transformed to the regular LQ problem for standard state space systems by restricted system equivalence transformation under some conditions. The system state is decomposed into free state and restricted state and the input is decomposed into free input 
and forced input. The optimal control is synthesized as state feedback. The optimal control-state pair is derived by solving an algebraic Riccati equation. All the finite poles of the closedloop system are located on the left-half complex plane.

The remainder of the paper is organized as follows. In Section 2, the indefinite LQ problem for irregular singular systems is transformed to the optimal problem for standard state space systems by restricted system equivalence transformation. In Section 3, we obtain the sufficient conditions for the unique existence of optimal control-state pair and solve the state feedback gain. A simulation example is exploited to demonstrate the effectiveness of the proposed results in Section 4. This paper is briefly concluded in Section 5.

Notation. Throughout the paper, the superscript " $T$ " stands for matrix transposition; $R^{n}$ denotes the $n$-dimensional Euclidean space; $R^{n \times m}$ is the set of $n \times m$ real matrices; $I_{n}$ is the $n \times n$ identity matrix; $\mathfrak{R} \lambda$ stands for the real part of $\lambda$; for real symmetric matrix $A, A>0$ means that $A$ is definitepositive and $A \geq 0$ means that $A$ is semidefinite-positive. The matrices, if not explicitly stated, are assumed to have compatible dimensions.

\section{Statement and Transformation of Indefinite LQ Problem}

Consider the optimal control problem of linear irregular singular systems

$$
E \dot{x}=A x+B u, \quad E x(0)=x_{0}
$$

with quadratic cost

$$
J(u, x)=\int_{0}^{\infty}\left[x^{T}, u^{T}\right] Q\left[x^{T}, u^{T}\right]^{T} d t
$$

where $E, A \in R^{m \times n}, B \in R^{m \times r}, Q \in R^{(n+r) \times(n+r)}, 0<\operatorname{rank} E=$ $p<n, Q^{T}=Q$, and $x, u$, and $x_{0}$ are state, input, and initial state, respectively.

System (1) is said to be regular if $m=n$ and $\operatorname{det}(s E-A) \not \equiv$ 0 ; otherwise, it is irregular. For the irregular singular system, Zhu et al. [13] discussed the state feedback LQ problem.

We denote the above LQ problem about (1) and (2) as problem $\mathbb{P}$ and define the set of admissible control-state pairs of problem $\mathbb{P}$ as

$$
\begin{array}{r}
\Omega=\{(u, x) \mid(u, x) \text { is piecewise continuous, } \\
\text { satisfies (1) and }|J(u, x)|<\infty\} .
\end{array}
$$

Problem $\mathbb{P}$ is called indefinite LQ problem if there are not only positive numbers but also negative numbers in the set of eigenvalues of matrix $Q$.

The control objective of this paper is to find an optimal control-state pair $\left(u^{*}, x^{*}\right) \in \Omega$ such that

$$
J\left(u^{*}, x^{*}\right)=\min _{(u, x) \in \Omega} J(u, x)
$$

when problem $\mathbb{P}$ is indefinite LQ problem.
Since rank $E=p$, there exist matrices $M_{11} \in R^{p \times m}$ and $N_{11} \in R^{n \times p}$ such that $M_{11} E N_{11}=I_{p}$, where $M_{11}$ has full row rank and $N_{11}$ full column rank. Suppose $M_{12}$ is one of the left zero divisors of $E$ and $N_{12}$ one of the right zero divisors of $E$; then the matrices $M_{1}=\left[M_{11}^{T}, M_{12}^{T}\right]^{T}$ and $N_{1}=\left[N_{11}, N_{12}\right]$ are nonsingular.

Let

$$
M_{1} A N_{1}=\left[\begin{array}{ll}
A_{11} & A_{12} \\
A_{21} & A_{22}
\end{array}\right], \quad M_{1} B=\left[\begin{array}{l}
B_{1} \\
B_{2}
\end{array}\right],
$$

where $A_{11} \in R^{p \times p}, B_{1} \in R^{p \times r}$.

Definition 1 (see [24]). System (1) is impulse controllable if there exists a smooth (impulse-free) control-state pair of system (1) for every initial condition.

Obviously, it is necessary for the solvability of the problem $\mathbb{P}$ that system (1) is impulse controllable. The following lemma establishes two necessary and sufficient conditions for the impulse controllability of system (1).

Lemma 2. System (1) is impulse controllable if and only if

$$
\operatorname{rank}\left[\begin{array}{lll}
A_{21} & A_{22} & B_{2}
\end{array}\right]=\operatorname{rank}\left[\begin{array}{ll}
A_{22} & B_{2}
\end{array}\right]
$$

or

$$
\operatorname{rank}\left[\begin{array}{ccc}
0 & E & 0 \\
E & A & B
\end{array}\right]=\operatorname{rank}\left[\begin{array}{ccc}
E & A & B
\end{array}\right]+\operatorname{rank} E .
$$

The proof is similar to that of Theorem 3 in [24], so it is omitted.

In the following discussions, we always assume that (7) holds.

Denote

$$
d=\operatorname{rank} A_{22}=\operatorname{rank}\left[\begin{array}{cc}
0 & E \\
E & A
\end{array}\right]-2 \operatorname{rank} E,
$$

and then there exist matrices $M_{21} \in R^{d \times(m-p)}$ and $N_{21} \epsilon$ $R^{(n-p) \times d}$ such that $M_{21} A_{22} N_{21}=I_{d}$, where $M_{21}$ has full row rank and $N_{21}$ full column rank. Suppose $M_{22}$ is one of the left zero divisors of $A_{22}$ and $N_{22}$ one of the right zero divisors of $A_{22}$; then the matrices $M_{2}=\left[M_{21}^{T}, M_{22}^{T}\right]^{T}$ and $N_{2}=$ $\left[N_{21}, N_{22}\right]$ are nonsingular.

Let

$$
\begin{gathered}
A_{12} N_{2}=\left[A_{121}, A_{122}\right], \quad M_{2} A_{21}=\left[\begin{array}{l}
A_{211} \\
A_{212}
\end{array}\right], \\
M_{2} B_{2}=\left[\begin{array}{l}
B_{21} \\
B_{22}
\end{array}\right],
\end{gathered}
$$

where $A_{121} \in R^{p \times d}, A_{211} \in R^{d \times p}$, and $B_{21} \in R^{d \times r}$.

Denote

$$
s=\operatorname{rank}\left[A_{22}, B_{2}\right]=\operatorname{rank}\left[\begin{array}{ccc}
0 & E & 0 \\
E & A & B
\end{array}\right]-2 \operatorname{rank} E,
$$

and then $\operatorname{rank}\left[A_{212}, B_{22}\right]=\operatorname{rank} B_{22}=s-d$ and there exist matrices $M_{31} \in R^{(s-d) \times(m-p-d)}$ and $N_{31} \in R^{r \times(s-d)}$ such that 
$M_{31} B_{22} N_{31}=I_{s-d}$, where $M_{31}$ has full row rank and $N_{31}$ full column rank. Suppose $M_{32}$ is one of the left zero divisors of $B_{22}$ and $N_{32}$ one of the right zero divisors of $B_{22}$; then the matrices $M_{3}=\left[M_{31}^{T}, M_{32}^{T}\right]^{T}$ and $N_{3}=\left[N_{31}, N_{32}\right]$ are nonsingular and $M_{32} A_{212}=0$.

Denote $N=\operatorname{diag}\left(\left[N_{1}, I_{r}\right]\right) \operatorname{diag}\left(\left[I_{p}, N_{2}, N_{3}\right]\right), M_{31} A_{212}=$ $A_{2121}, B_{1} N_{3}=\left[B_{11}, B_{12}\right], B_{21} N_{3}=\left[B_{211}, B_{212}\right]$, and

$$
\left[x_{1}^{T}, x_{2}^{T}, x_{3}^{T}, u_{1}^{T}, u_{2}^{T}\right]^{T}=N^{-1}\left[x^{T}, u^{T}\right]^{T}
$$

where $B_{11} \in R^{p \times(s-d)}, B_{211} \in R^{d \times(s-d)}, x_{1} \in R^{p}, x_{2} \in R^{d}$, $x_{3} \in R^{n-p-d}, u_{1} \in R^{s-d}$, and $u_{2} \in R^{r+d-s}$; then the system (1) is restricted system equivalent (r.s.e.) to the following system:

$$
\begin{gathered}
\dot{x}_{1}=\bar{A}_{11} x_{1}+A_{122} x_{3}+\bar{B}_{12} u_{2}, \quad x_{1}(0)=M_{11} x_{0}, \\
x_{2}=\bar{A}_{21} x_{1}-B_{212} u_{2} \\
u_{1}=\bar{A}_{31} x_{1},
\end{gathered}
$$

where $\bar{A}_{11}=A_{11}-A_{121} A_{211}+A_{121} B_{211} A_{2121}-B_{11} A_{2121}, \bar{B}_{12}=$ $B_{12}-A_{121} B_{212}, \bar{A}_{21}=B_{211} A_{2121}-A_{211}$, and $\bar{A}_{31}=-A_{2121}$.

In the system (12), the state variables $x_{1}, x_{2}$ and input variable $u_{1}$ are determined uniquely by $x_{3}$ and $u_{2}$. Thus the state variable $x_{3}$ is free and the input variable $u_{1}$ is not free. We call $x_{3}$ the free state and $u_{1}$ the forced input. Accordingly, $x_{1}$ and $x_{2}$ are called restricted state and $u_{2}$ is called free input. When $\bar{B}_{12}=0$ and $B_{212}=0$, system (12) has a unique solution if and only if $A_{122}=0$ or the dimension of $x_{3}$ is zero.

A system is usually required to have a unique solution when it is handled. The following lemma establishes a necessary and sufficient condition for the uniqueness of solutions of system (1).

Lemma 3. System (1) with $B=0$ has a unique solution if and only if

$$
\operatorname{rank}\left[\begin{array}{cc}
0 & E \\
E & A
\end{array}\right]=\operatorname{rank}\left[\begin{array}{c}
E \\
A
\end{array}\right]+\operatorname{rank} E
$$

or

$$
\operatorname{rank}\left[\begin{array}{cc}
0 & E \\
E & A
\end{array}\right]=n+\operatorname{rank} E
$$

In the following discussions, we always assume that (13) or (14) holds; that is, we consider the control problem of the following system:

$$
\begin{gathered}
\dot{x}_{1}=\bar{A}_{11} x_{1}+\bar{B}_{12} u_{2}, \quad x_{1}(0)=M_{11} x_{0}, \\
x_{2}=\bar{A}_{21} x_{1}-B_{212} u_{2}, \\
u_{1}=\bar{A}_{31} x_{1} .
\end{gathered}
$$

We have the following two conclusions about the input of system (1).

Lemma 4. System (1) has no forced input if and only if

$$
\operatorname{rank}\left[\begin{array}{cc}
0 & E \\
E & A
\end{array}\right]=\operatorname{rank}\left[\begin{array}{ccc}
0 & E & 0 \\
E & A & B
\end{array}\right] .
$$

Lemma 5. If the equality

$$
\operatorname{rank}\left[\begin{array}{ccc}
0 & E & 0 \\
E & A & B
\end{array}\right]=r+\operatorname{rank}\left[\begin{array}{cc}
0 & E \\
E & A
\end{array}\right]
$$

holds, then system (1) has no free input.

It is meaningless to design the controller for a system without free input. In the following discussions, we always assume that (17) does not hold; that is, the system (1) satisfies that

$$
\operatorname{rank}\left[\begin{array}{ccc}
0 & E & 0 \\
E & A & B
\end{array}\right]<r+\operatorname{rank}\left[\begin{array}{cc}
0 & E \\
E & A
\end{array}\right]
$$

Denote

$$
\begin{aligned}
& P_{1}=N_{12} N_{21} M_{21} M_{12} \\
& P_{2}=N_{31} M_{31} M_{22} M_{12} \text {, } \\
& P_{3}=\left[P_{31}, P_{32}, P_{33}\right] \\
& =\left[\begin{array}{ccc}
N_{11}-P_{1} A N_{11}+P_{1} B P_{2} A N_{11} & N_{12} N_{22} & -P_{1} B N_{32} \\
-P_{2} A N_{11} & 0 & N_{32}
\end{array}\right], \\
& N_{4}=\left[\begin{array}{ccc}
I_{p} & 0 & 0 \\
\bar{A}_{21} & 0 & -B_{212} \\
0 & I_{n-p-d} & 0 \\
\bar{A}_{31} & 0 & 0 \\
0 & 0 & I_{r+d-s}
\end{array}\right],
\end{aligned}
$$

and $\bar{Q}=\left(\bar{Q}_{i j}\right)_{3 \times 3}=P_{3}^{T} Q P_{3}$, where $\bar{Q}_{11} \in R^{p \times p}, \bar{Q}_{22} \in$ $R^{(n-p-d) \times(n-p-d)}$, and $\bar{Q}_{33} \in R^{(r+d-s) \times(r+d-s)}$; then $P_{3}=N N_{4}$ and the quadratic cost corresponding to system (15) is

$$
\begin{aligned}
& J_{1}\left(u_{2}, x_{1}, x_{3}\right) \\
& =\int_{0}^{\infty}\left[x_{1}^{T}, x_{3}^{T}, u_{2}^{T}\right] N_{4}^{T} N^{T} Q_{N}\left[x_{1}^{T}, x_{3}^{T}, u_{2}^{T}\right]^{T} d t \\
& =\int_{0}^{\infty}\left(x_{1}^{T} \bar{Q}_{11} x_{1}+x_{3}^{T} \bar{Q}_{22} x_{3}+u_{2}^{T} \bar{Q}_{33} u_{2}\right. \\
& \left.\quad+2 x_{1}^{T} \bar{Q}_{12} x_{3}+2 x_{1}^{T} \bar{Q}_{13} u_{2}+2 x_{3}^{T} \bar{Q}_{23} u_{2}\right) d t .
\end{aligned}
$$

Theorem 6. If there exist matrices $M_{11} \in R^{p \times m}, M_{12} \in$ $R^{(m-p) \times m}, M_{21} \in R^{d \times(m-p)}, M_{22} \in R^{(m-p-d) \times(m-p)}, M_{31} \in$ $R^{(s-d) \times(m-n)}, N_{11} \in R^{n \times p}, N_{12} \in R^{n \times(n-p)}, N_{21} \in R^{(n-p) \times d}$, $N_{22} \in R^{(n-p) \times(n-p-d)}, N_{31} \in R^{r \times(p+s-n)}$, and $N_{32} \in R^{r \times(n+r-p-s)}$ satisfying the following

(1) $M_{11}, M_{12}, M_{21}, M_{22}$, and $M_{31}$ have full row rank and $N_{11}, N_{12}, N_{21}, N_{22}, N_{31}$, and $N_{32}$ have full column rank,

(2) $M_{11} E N_{11}=I_{p}, M_{12} E=0, E N_{12}=0, M_{22} M_{12} A N_{12}=$ 0, $M_{31} M_{22} M_{12} B N_{31}=I_{p+s-n}, M_{21} M_{12} A N_{12} N_{21}=I_{d}$, $M_{12} A N_{12} N_{22}=0, M_{22} M_{12} B N_{32}=0$,

(3) $M_{11}\left(B-A P_{1} B\right) N_{32} \neq 0$, 
(4) $P_{32}^{T} Q P_{3}=0$,

(5) $P_{33}^{T} Q P_{33}>0$,

(6) $\left[P_{31}, P_{33}\right]^{T} \mathrm{Q}\left[P_{31}, P_{33}\right] \geq 0$,

then problem $\mathbb{P}$ can be transformed to a regular LQ problem of the standard state space system.

Proof. When $P_{32}^{T} Q P_{3}=0$ holds, $\bar{Q}_{12}=0, \bar{Q}_{22}=0$, and $\bar{Q}_{23}=$ 0 , and the quadratic cost is

$$
\begin{aligned}
J_{2}\left(u_{2}, x_{1}\right) & =\int_{0}^{\infty}\left(x_{1}^{T} \bar{Q}_{11} x_{1}+2 x_{1}^{T} \bar{Q}_{13} u_{2}+u_{2}^{T} \bar{Q}_{33} u_{2}\right) d t \\
& =\int_{0}^{\infty}\left[x_{1}^{T}, u_{2}^{T}\right]\left[P_{31}, P_{33}\right]^{T} Q\left[P_{31}, P_{33}\right]\left[x_{1}^{T}, u_{2}^{T}\right]^{T} d t
\end{aligned}
$$

Therefore, when $\left[P_{31}, P_{33}\right]^{T} Q\left[P_{31}, P_{33}\right] \geq 0$, the weighted matrix of $x_{1}$ and $u_{2}$ in the objective function is semidefinitepositive. $P_{33}^{T} Q P_{33}>0$ ensures that the optimal control problem of system (15) with cost (21) is a regular LQ problem. In addition, $M_{11}\left(B-A P_{1} B\right) N_{32} \neq 0$, which is equivalent to $\bar{B}_{12} \neq 0$, constitutes the necessary and sufficient condition of existing free input in system (15) with (18).

When the conditions in Theorem 6 are satisfied, let

$$
\bar{u}_{2}=u_{2}+\bar{Q}_{33}^{-1} \bar{Q}_{13}^{T} x_{1} \text {, }
$$

and then system (15) can be written as

$$
\begin{gathered}
\dot{x}_{1}=\widetilde{A}_{11} x_{1}+\bar{B}_{12} \bar{u}_{2}, \quad x_{1}(0)=M_{11} x_{0} \\
x_{2}=\widetilde{A}_{21} x_{1}-B_{212} \bar{u}_{2} \\
u_{1}=\bar{A}_{31} x_{1}
\end{gathered}
$$

and the corresponding quadratic cost is

$$
J_{3}\left(\bar{u}_{2}, x_{1}\right)=\int_{0}^{\infty}\left(x_{1}^{T} \widetilde{Q}_{11} x_{1}+\bar{u}_{2}^{T} \bar{Q}_{33} \bar{u}_{2}\right) d t
$$

where $\widetilde{A}_{11}=\bar{A}_{11}-\bar{B}_{12} \bar{Q}_{33}^{-1} \bar{Q}_{13}^{T}, \widetilde{A}_{21}=\bar{A}_{21}+B_{212} \bar{Q}_{33}^{-1} \bar{Q}_{13}^{T}$, and $\widetilde{\mathrm{Q}}_{11}=\overline{\mathrm{Q}}_{11}-\overline{\mathrm{Q}}_{13} \overline{\mathrm{Q}}_{33}^{-1} \overline{\mathrm{Q}}_{13}^{T}$.

We denote $\mathbb{P}_{1}$ as the optimal control problem of system (23) with cost (24) and define the set of admissible controlstate pairs of problem $\mathbb{P}_{1}$ as

$\Omega_{1}$

$$
\begin{array}{r}
=\left\{\left(u_{1}, \bar{u}_{2}, x_{1}, x_{2}\right) \mid\left(u_{1}, \bar{u}_{2}, x_{1}, x_{2}\right)\right. \text { is piecewise continuous, } \\
\text { satisfies } \left.(23) \text { and }\left|J_{3}\left(\bar{u}_{2}, x_{1}\right)\right|<\infty\right\} .
\end{array}
$$

According to the definition of an equivalent relation of the two optimal control problems [12], problem $\mathbb{P}$ is equivalent to problem $\mathbb{P}_{1}$. Obviously, $\mathbb{P}_{1}$ is an optimal problem of the standard state space system.

\section{Design of the State Feedback Controller}

In this section, we solve the problem $\mathbb{P}$ via solving $\mathbb{P}_{1}$.

Before further discussion, we first give two lemmas.

Lemma 7. The pair $\left(\widetilde{A}_{11}, \bar{B}_{12}\right)$ is stabilizable if and only if $\operatorname{rank}[\lambda E-A, B]=\operatorname{rank}\left[\begin{array}{ccc}0 & E & 0 \\ E & A & B\end{array}\right]-\operatorname{rank} E, \quad \forall \lambda, \mathfrak{R} \lambda \geq 0$.

Proof. Denote

$M=\left[\begin{array}{cccc}I_{p} & -A_{121} & -B_{11} & 0 \\ 0 & I_{d} & -B_{211} & 0 \\ 0 & 0 & I_{s-d} & 0 \\ 0 & 0 & 0 & I_{m-p-s}\end{array}\right]\left[\begin{array}{cc}I_{p+d} & 0 \\ 0 & M_{3}\end{array}\right]\left[\begin{array}{cc}I_{p} & 0 \\ 0 & M_{2}\end{array}\right] M_{1}$

then

$$
M[A, B] N=\left[\begin{array}{ccccc}
\bar{A}_{11} & 0 & 0 & 0 & \bar{B}_{12} \\
-\bar{A}_{21} & I_{d} & 0 & 0 & B_{212} \\
A_{2121} & 0 & 0 & I_{s-d} & 0 \\
0 & 0 & 0 & 0 & 0
\end{array}\right]
$$

The pair $\left(\widetilde{A}_{11}, \bar{B}_{12}\right)$ is stabilizable if and only if

$$
\operatorname{rank}\left[\lambda I-\widetilde{A}_{11}, \bar{B}_{12}\right]=p, \quad \forall \lambda, \mathfrak{R} \lambda \geq 0 ;
$$

that is

$$
\operatorname{rank}\left[\lambda I-\bar{A}_{11}, \bar{B}_{12}\right]=p, \quad \forall \lambda, \mathfrak{R} \lambda \geq 0,
$$

which is equivalent to (26) from (28).

Denote $Q=\left[Q_{1}, Q_{2}\right]$, where $Q_{1} \in R^{(n+r) \times n}, Q_{2} \in R^{(n+r) \times r}$.

Lemma 8. When the conditions in Theorem 6 are satisfied, the $\operatorname{pair}\left(\widetilde{A}_{11}, \widetilde{Q}_{11}\right)$ is detectable if and only if

$$
\operatorname{rank}\left[\begin{array}{cc}
A-\lambda E & B \\
P_{3}^{T} Q_{1} & P_{3}^{T} Q_{2}
\end{array}\right]=n+r, \quad \forall \lambda, \Re \lambda \geq 0 .
$$

Proof. Denote $\widehat{Q}=\left[\widehat{Q}_{1}, \widehat{Q}_{2}, \widehat{Q}_{3}, \widehat{Q}_{4}, \widehat{Q}_{5}\right]=N^{T} Q N$, where $\widehat{Q}_{1} \in R^{(n+r) \times p}, \widehat{Q}_{2} \in R^{(n+r) \times d}, \widehat{Q}_{3} \in R^{(n+r) \times(n-p-d)}, \widehat{Q}_{4} \in$ $R^{(n+r) \times(s-d)}$, and $\widehat{Q}_{5} \in R^{(n+r) \times(r+d-s)}$; then $\bar{Q}=N_{4}^{T} \widehat{Q} N_{4}$ and $N_{4}^{T} \widehat{Q}_{3}=P_{3}^{T} Q_{1} N_{12} N_{22}=0$.

The pair $\left(\widetilde{A}_{11}, \widetilde{Q}_{11}\right)$ is detectable if and only if

$$
\operatorname{rank}\left[\begin{array}{c}
\widetilde{A}_{11}-\lambda I_{p} \\
\widetilde{Q}_{11}
\end{array}\right]=p, \quad \forall \lambda, \Re \lambda \geq 0
$$

which is equivalent to

$$
\operatorname{rank}\left[\begin{array}{cc}
\bar{A}_{11}-\lambda I_{p} & \bar{B}_{12} \\
\bar{Q}_{11} & \bar{Q}_{13} \\
\bar{Q}_{13}^{T} & \bar{Q}_{33}
\end{array}\right]=n+r-s, \quad \forall \lambda, \Re \lambda \geq 0
$$


that is,

$$
\begin{array}{r}
\operatorname{rank}\left[\begin{array}{cccc}
A_{11}-\lambda I_{p} & A_{121} & B_{11} & B_{12} \\
A_{211} & I_{d} & B_{211} & B_{212} \\
A_{2121} & 0 & I_{s-d} & 0 \\
N_{4}^{T} \widehat{Q}_{1} & N_{4}^{T} \widehat{Q}_{2} & N_{4}^{T} \widehat{Q}_{4} & N_{4}^{T} \widehat{Q}_{5}
\end{array}\right]=n+r, \\
\forall \lambda, \Re \lambda \geq 0 .
\end{array}
$$

An easy computation shows that the above equality is equivalent to (31).

Definition 9 (see [6]). The finite $\lambda$ s satisfying $\operatorname{det}(\lambda E-A)=0$ are called finite poles for the singular system $E \dot{x}=A x$.

In the following, we give the conclusion concerning problem $\mathbb{P}$.

Theorem 10. Assume the conditions in Theorem 6 are satisfied and the rank equalities (26) and (31) hold; then problem $\mathbb{P}$ has a unique optimal control-state pair and the optimal control can be synthesized as state feedback. The finite poles of closed-loop system all are located on the left-half complex plane and the optimal value is $J^{*}=x_{0}^{T} M_{11}^{T} P M_{11} x_{0}$, where $P$ is the unique semidefinite-positive solution of the Riccati equation

$$
P \widetilde{A}_{11}+\widetilde{A}_{11}^{T} P-P \bar{B}_{12} \bar{Q}_{33}^{-1} \bar{B}_{12}^{T} P+\widetilde{Q}_{11}=0 .
$$

Proof. Since the pair $\left(\widetilde{A}_{11}, \bar{B}_{12}\right)$ is stabilizable and the pair $\left(\widetilde{A}_{11}, \widetilde{Q}_{11}\right)$ is detectable, Riccati equation (35) has a unique semidefinite-positive $P$ and the eigenvalues of matrix $\widetilde{A}_{11}-$ $\bar{B}_{12} \bar{Q}_{33}^{-1} \bar{B}_{12}^{T} P$ are located on the left-half complex plane.

According to Maximum Principle, the unique optimal control-state pair $\left(u_{1}^{*}, \bar{u}_{2}^{*}, x_{1}^{*}, x_{2}^{*}\right)$ of the optimal control problem of system (23) with cost (24) is

$$
\left[\begin{array}{c}
x_{1}^{*} \\
x_{2}^{*} \\
u_{1}^{*} \\
\bar{u}_{2}^{*}
\end{array}\right]=\left[\begin{array}{c}
I_{p} \\
\widetilde{A}_{21}+B_{212} \bar{Q}_{33}^{1} \bar{B}_{12}^{T} P \\
\bar{A}_{31} \\
-\bar{Q}_{33}^{1} \bar{B}_{12}^{T} P
\end{array}\right] x_{1}^{*},
$$

and the optimal value is $J^{*}=x_{0}^{T} M_{11}^{T} P M_{11} x_{0}$, where $x_{1}^{*}$ is the solution of equation

$$
\dot{x}_{1}=\left(\widetilde{A}_{11}-\bar{B}_{12} \bar{Q}_{33}^{-1} \bar{B}_{12}^{T} P\right) x_{1}, \quad x_{1}(0)=M_{11} x_{0} .
$$

It follows from (11) and (22) that the unique optimal control-state pair $\left(u^{*}, x^{*}\right)$ of problem $\mathbb{P}$ is

$$
\left[\begin{array}{l}
x^{*} \\
u^{*}
\end{array}\right]=N\left[\begin{array}{c}
I_{p} \\
\widetilde{A}_{21}+B_{212} \bar{Q}_{33}^{-1} \bar{B}_{12}^{T} P \\
\bar{A}_{31} \\
-\bar{Q}_{33}^{-1}\left(\bar{Q}_{13}^{T}+\bar{B}_{12}^{T} P\right)
\end{array}\right] x_{1}^{*}
$$

and the optimal closed-loop system is

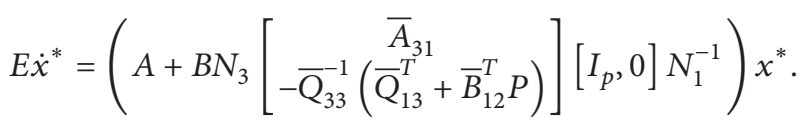

An easy computation shows that the finite poles of optimal closed-loop system (39) and the eigenvalues of matrix $\widetilde{A}_{11}-\bar{B}_{12} \bar{Q}_{33}^{-1} \bar{B}_{12}^{T} P$ are the same. Therefore, the finite eigenvalues of closed-loop system all are located on the lefthalf complex plane.

\section{A Simulation Example}

In this section, we give a simple example to illuminate the design method of state feedback controller and demonstrate its feasibility.

Consider the irregular singular system (1) with quadratic cost (2), where

$$
\begin{aligned}
& E=\left[\begin{array}{llll}
3 & 1 & 2 & 2 \\
0 & 2 & 1 & 1 \\
0 & 2 & 1 & 1 \\
1 & 3 & 2 & 2 \\
1 & 1 & 1 & 1
\end{array}\right], \quad A=\left[\begin{array}{llll}
1 & 0 & 2 & 5 \\
5 & 4 & 3 & 1 \\
2 & 3 & 6 & 4 \\
4 & 6 & 6 & 2 \\
1 & 5 & 4 & 5
\end{array}\right], \\
& B=\left[\begin{array}{lll}
3 & 1 & 6 \\
6 & 2 & 6 \\
3 & 5 & 4 \\
3 & 1 & 5 \\
5 & 2 & 4
\end{array}\right], \quad x_{0}=\left[\begin{array}{c}
-1 \\
1 \\
1 \\
1 \\
0
\end{array}\right] \text {, } \\
& Q=\left[\begin{array}{ccccccc}
3 & 2 & 1 & 2 & 1 & 3 & 2 \\
2 & 4 & 0 & 0 & -2 & 2 & -1 \\
1 & 0 & 2 & -2 & 2 & 0 & -1 \\
2 & 0 & -2 & 4 & -1 & -1 & -1 \\
1 & -2 & 2 & -1 & 5 & -2 & -1 \\
3 & 2 & 0 & -1 & -2 & 4 & -2 \\
2 & -1 & -1 & -1 & -1 & -2 & 1
\end{array}\right]
\end{aligned}
$$

Obviously, $p=2, d=2$, and $s=3$ and the eigenvalues of $Q$ are $-4.0310,0.3589,2.0810,3.1335,5.2966,6.6965,9.4645$. Let

$$
\begin{aligned}
& M_{1}=\left[\begin{array}{ccccc}
0 & -0.5 & 0 & 0 & 1 \\
0 & 0.5 & 0 & 0 & 0 \\
0 & -1 & 1 & 0 & 0 \\
0 & -1 & 0 & 1 & -1 \\
1 & 1 & 0 & 0-3 &
\end{array}\right] \\
& N_{1}=\left[\begin{array}{cccc}
1 & 0 & -0.5 & -0.5 \\
0 & 1 & -0.5 & -0.5 \\
0 & 0 & 1 & 0 \\
0 & 0 & 0 & 1
\end{array}\right] \text {, } \\
& M_{2}=\left[\begin{array}{ccc}
0.2 & 0 & 0 \\
0.1 & -\frac{1}{3} & 0 \\
0.8 & -\frac{2}{3} & 1
\end{array}\right], \quad N_{21}=\left[\begin{array}{cc}
1 & -1 \\
0 & 1
\end{array}\right] \text {, } \\
& M_{3}=-\frac{15}{46}, \quad N_{3}=\left[\begin{array}{ccc}
1 & \frac{21}{46} & \frac{13}{23} \\
0 & 1 & 0 \\
0 & 0 & 1
\end{array}\right] \text {, }
\end{aligned}
$$



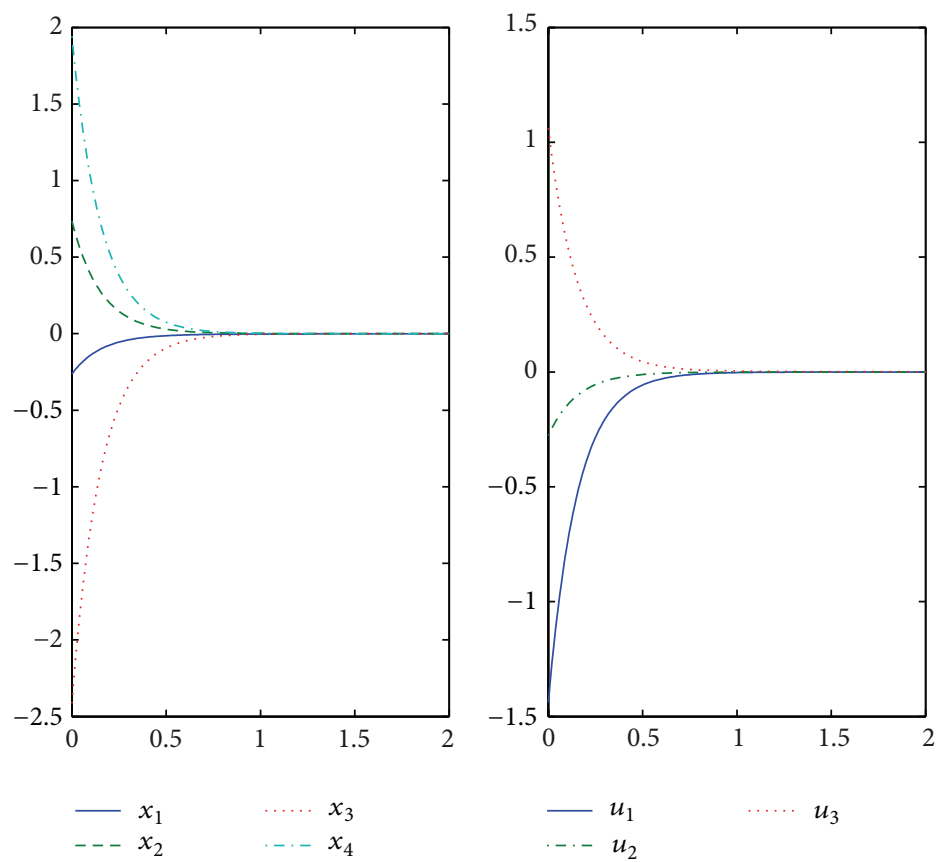

FIGURE 1: Curves of optimal control-state pair.

then the system (1) is r.s.e. to the following system:

$$
\begin{gathered}
\dot{x}_{1}=\left[\begin{array}{cc}
-0.3154 & 1.9837 \\
0.2826 & 5.2320
\end{array}\right] x_{1}+\left[\begin{array}{cc}
-3.4185 & -2.1848 \\
4.9946 & 6.9457
\end{array}\right] \bar{u}_{2}, \\
x_{1}(0)=\left[\begin{array}{c}
-0.5 \\
0.5
\end{array}\right] \\
x_{2}=\left[\begin{array}{cc}
0.5526 & 0.5796 \\
0.3102 & -0.5298
\end{array}\right] x_{1}-\left[\begin{array}{cc}
0.3261 & -0.7391 \\
2.3804 & 2.8043
\end{array}\right] \bar{u}_{2} \\
u_{1}=[0.6304,-3.1957] x_{1}
\end{gathered}
$$

and the corresponding quadratic cost is

$$
\begin{aligned}
J_{1}\left(\bar{u}_{2}, x_{1}\right)=\int_{0}^{\infty}( & x_{1}^{T}\left[\begin{array}{cc}
1.8721 & -4.4330 \\
-4.4330 & 28.3724
\end{array}\right] x_{1} \\
& \left.+\bar{u}_{2}^{T}\left[\begin{array}{cc}
65.4607 & 77.0631 \\
77.0631 & 110.1961
\end{array}\right] \bar{u}_{2}\right) d t
\end{aligned}
$$

under the transformations (11) and (22).

The unique positive semidefinite solution of Riccati equation (35) is

$$
P=\left[\begin{array}{cc}
1.8835 & 2.0224 \\
2.0224 & 28.1168
\end{array}\right],
$$

the optimal performance index is $J^{*}=6.4889$, and the simulation results are displayed in Figure 1.

\section{Conclusion}

In this paper the indefinite LQ problem for irregular singular systems has been investigated. By restricted system equivalence transformation, we transformed the indefinite LQ problem for irregular singular systems to the regular LQ problem for standard state space systems. System state was decomposed into free state and restricted state and input was decomposed into free input and forced input. Based on optimization theory for standard state space systems, we have derived optimal control-state pair by solving an algebraic Riccati equation and the optimal control was synthesized as state feedback under some general conditions. The finite poles of closed-loop system are all located on the left-half complex plane.

However, there are many problems unsolved about the indefinite LQ problem of the irregular singular systems. For example, in this paper the effect of external disturbances on the system has not been involved. More importantly, in the controller design, we need to solve an algebraic Riccati equation, which is still a challenge. Therefore, we think that the significance of the paper exists in theory more than in practice.

\section{Conflict of Interests}

The authors declare that there is no conflict of interests regarding the publication of this paper.

\section{Acknowledgments}

This work was partially supported by National Natural Science Foundation of China under Contract no. 11131006 and Natural Science Foundation of Zhejiang Province under Grant Y1110036. 


\section{References}

[1] D. J. Hill and I. M. Mareels, "Stability theory for differential/ algebraic systems with application to power systems," IEEE Transactions on Circuits and Systems, vol. 37, no. 11, pp. 14161423, 1990.

[2] D. G. Luenberger, "Singular dynamic leontief systems," Econometrics, vol. 45, pp. 991-995, 1977.

[3] S. S. Sastry and C. A. Desoer, "Jump behavior of circuits and systems," IEEE Transactions on Circuits and Systems, vol. 28, no. 12, pp. 1109-1124, 1981.

[4] J. Y. Ishihara, M. H. Terra, and R. M. Sales, "The full information and state feedback $\mathrm{H}_{2}$ optimal controllers for descriptor systems," Automatica, vol. 39, no. 3, pp. 391-402, 2003.

[5] S. L. Campbell, Singular System of Differential Equations II, Pitman, San Francisco, Calif, USA, 1982.

[6] L. Dai, Singular Control Systems, vol. 118 of Lecture Notes in Co ntrol and Information Sciences, Springer, New York, NY, USA, 1989.

[7] D. Wang and C. B. Soh, "On regularizing singular systems by decentralized output feedback," IEEE Transactions on Automatic Control, vol. 44, no. 1, pp. 148-152, 1999.

[8] S. Xu, J. Lam, Y. Zou, and J. Li, "Robust admissibility of time-varying singular systems with commensurate time delays," Automatica, vol. 45, no. 11, pp. 2714-2717, 2009.

[9] R. E. Kalman, "Contributions to the theory of optimal control," Boletin de la Socieda d Matematica Mexica, vol. 5, pp. 102-119, 1960.

[10] B. D. O. Anderson and J. B. Moore, Optimal Control: Linear Quadratic Methods, Prentice-Hall, Englewood Cliffs, NJ, USA, 1989.

[11] D. J. Bender and A. J. Laub, "The linear-quadratic optimal regulator for descriptor systems," IEEE Transactions on Automatic Control, vol. 32, no. 8, pp. 672-688, 1987.

[12] Z. L. Cheng, H. M. Hong, and J. F. Zhang, "The optimal regulation of generalized state-space systems with quadratic cost," Automatica, vol. 24, no. 5, pp. 707-710, 1988.

[13] J. Zhu, S. Ma, and Z. Cheng, "Singular LQ problem for nonregular descriptor systems," IEEE Transactions on Automatic Control, vol. 47, no. 7, pp. 1128-1133, 2002.

[14] Y. C. You, "Optimal control for linear system with quadratic indefinite criterion on Hilbert spaces," Chinese Annals of Mathematics, Series B, vol. 4, no. 1, pp. 21-32, 1983.

[15] S. Chen, X. Li, and X. Y. Zhou, "Stochastic linear quadratic regulators with indefinite control weight costs," SIAM Journal on Control and Optimization, vol. 36, no. 5, pp. 1685-1702, 1998.

[16] J. C. Engwerda, "The indefinite LQ-problem: the finite planning horizon case," in Proceedings of the 5th International Symposium on Dynamic Games and Applications, pp. 1-18, 1992.

[17] S. Bittanti, A. J. Laub, and J. C. Willems, The Riccati Equation, Springer, Berlin, Germany, 1991.

[18] D. J. Clements and B. D. Anderson, Singular Optimal Control: The Linear Quadratic Problem, Springer, Berlin, Germany, 1978.

[19] H. L. Trentelman, "The regular free-endpoint linear quadratic problem with indefinite cost," SIAM Journal on Control and Optimization, vol. 27, no. 1, pp. 27-42, 1989.

[20] A. C. Ran and H. L. Trentelman, "Linear quadratic problems with indefinite cost for discrete time systems," SIAM Journal on Matrix Analysis and Applications, vol. 14, no. 3, pp. 776-797, 1993.
[21] F. Bucci and L. Pandolfi, "The regulator problem with indefinite quadratic cost for boundary control systems: the finite horizon case," Systems \& Control Letters, vol. 39, no. 2, pp. 79-86, 2000.

[22] P. Cui and C.-H. Zhang, "Indefinite linear quadratic optimal control problem for singular linear discrete-time system: krein space method," Acta Automatica Sinica, vol. 33, no. 6, pp. 635640, 2007.

[23] P. Cui, C. Zhang, H. Zhang, and H. Zhao, "Indefinite linear quadratic optimal control problem for singular discrete-time system with multiple input delays," Automatica, vol. 45, no. 10, pp. 2458-2461, 2009.

[24] J. Y. Ishihara and M. H. Terra, "Impulse controllability and observability of rectangular descriptor systems," IEEE Transactions on Automatic Control, vol. 46, no. 6, pp. 991-994, 2001. 


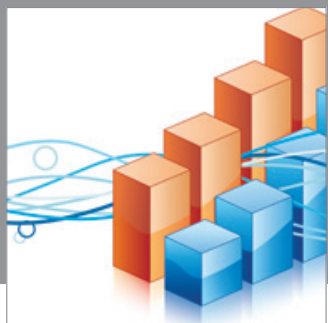

Advances in

Operations Research

mansans

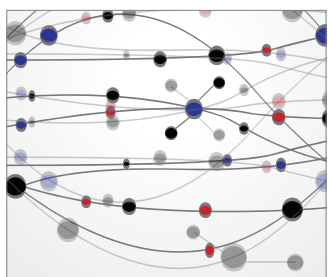

The Scientific World Journal
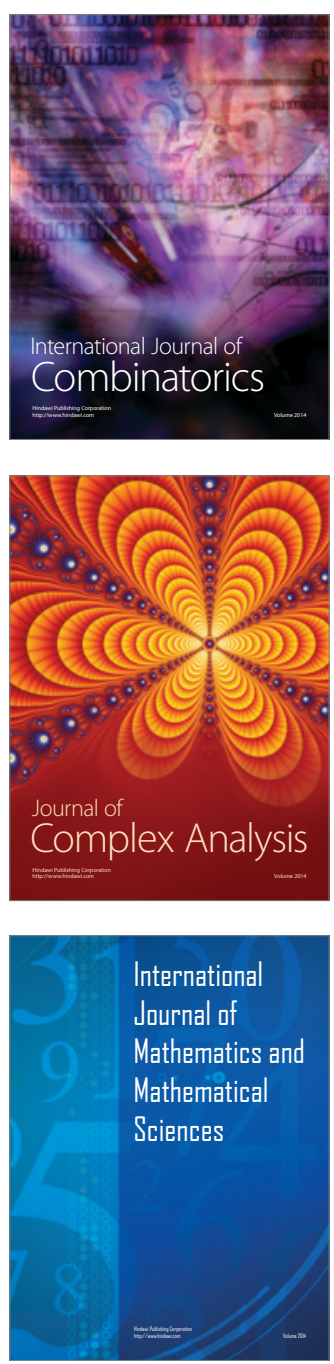
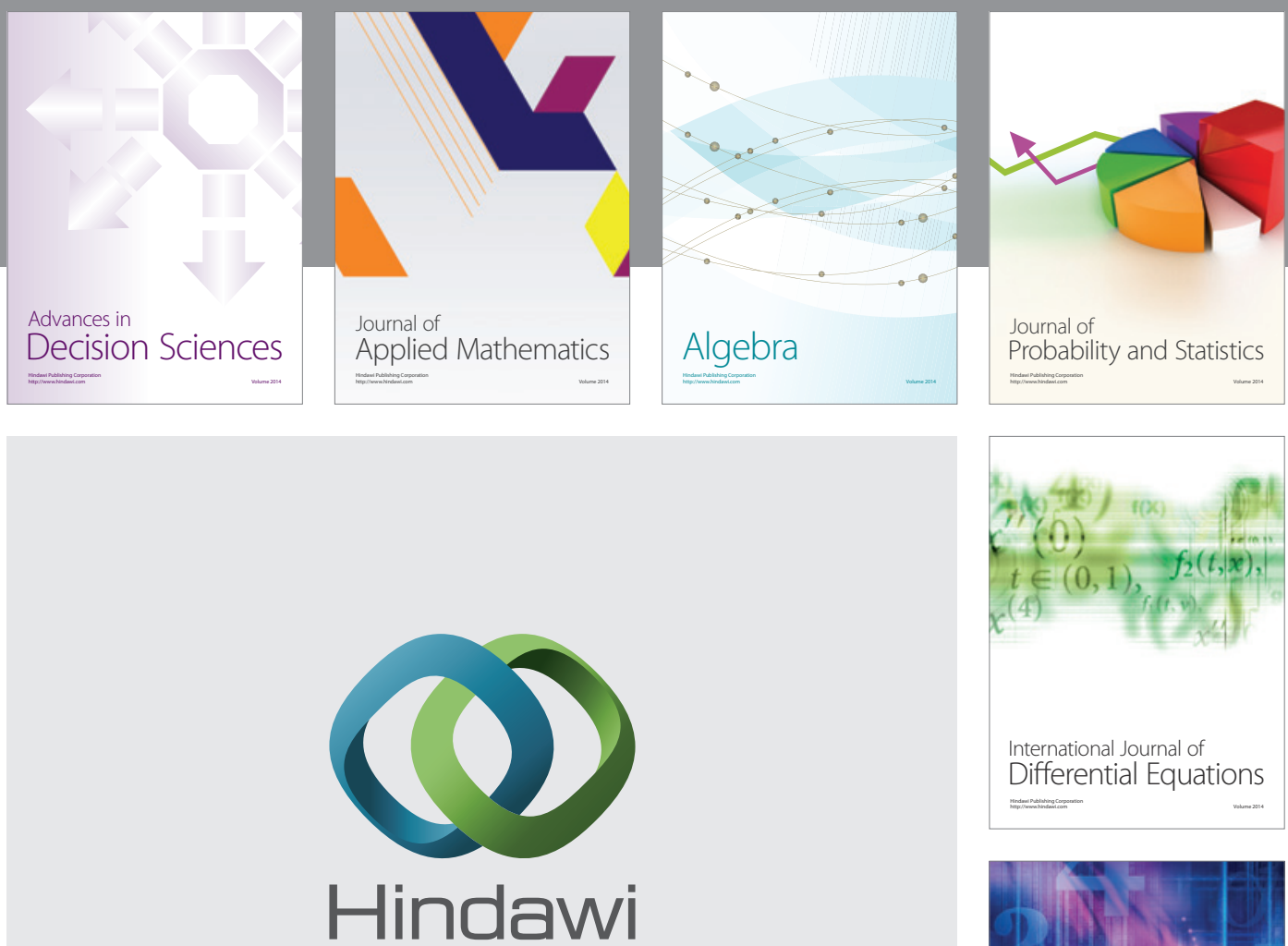

Submit your manuscripts at http://www.hindawi.com
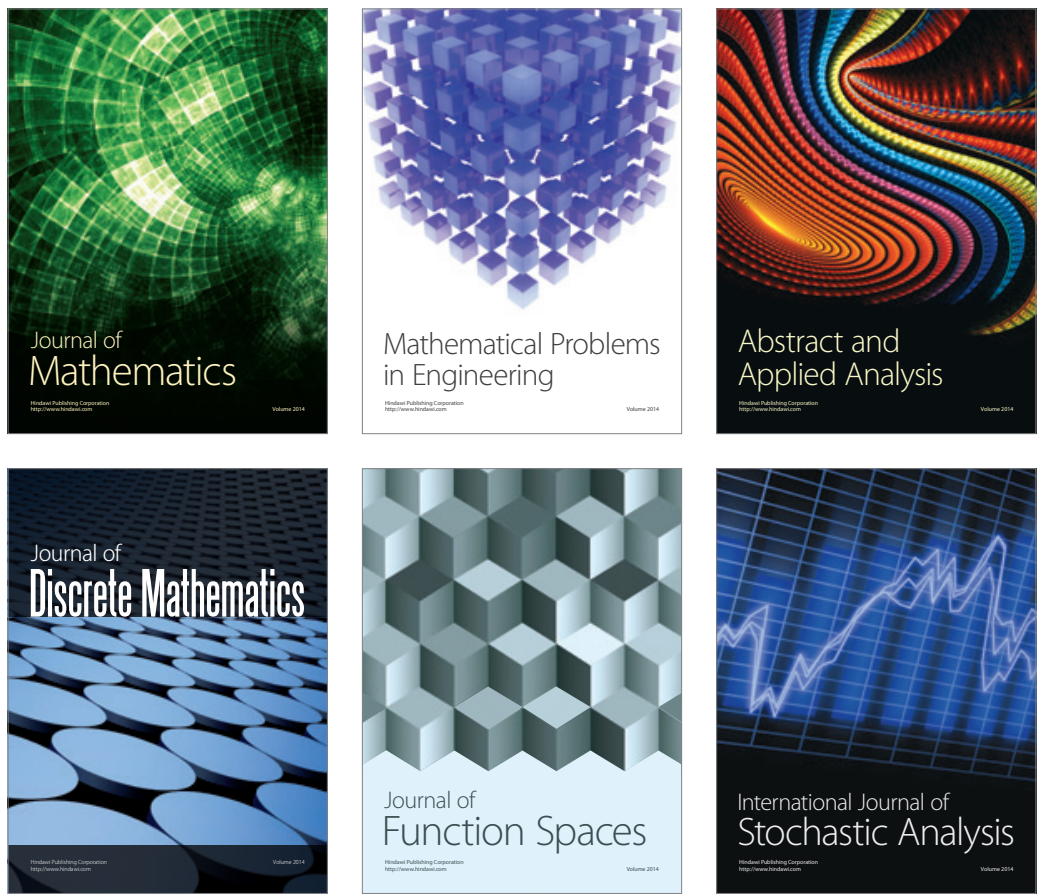

Journal of

Function Spaces

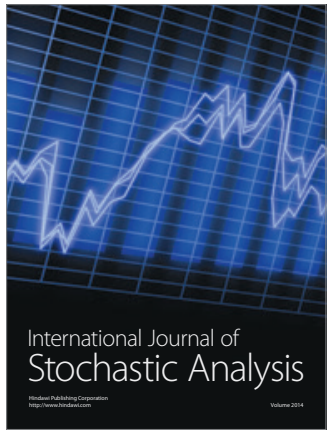

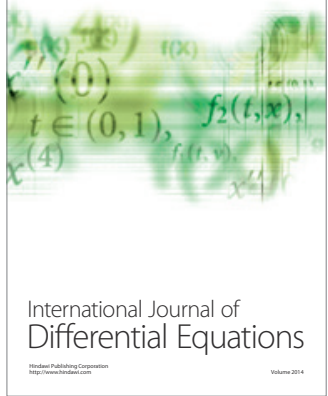
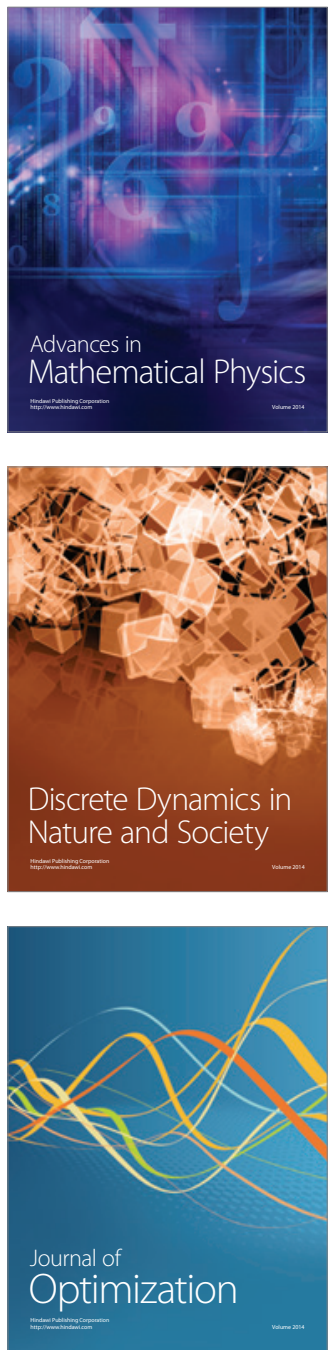